\title{
Importance of Imaging Procedures in the Evaluation of Methotrexate-Associated Lymphoproliferative Disorder of the Thyroid Gland: A Case Study
}

\author{
Mitsuaki Tokumaru ${ }^{a}$ Kenji Ohba ${ }^{a}$ b Kennichi Kakudo ${ }^{c}$ Takafumi Suda $^{b}$ \\ Yutaka Oki $^{\mathrm{d}}$ Yoshie Goto ${ }^{\mathrm{a}}$

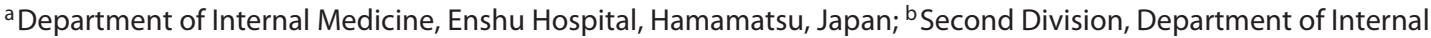 \\ Medicine, Hamamatsu University School of Medicine, Hamamatsu, Japan; ' Department of Pathology and Thyroid \\ Disease Center, Izumi City General Hospital, Izumi, Japan; ${ }^{d}$ Department of Family and Community Medicine, \\ Hamamatsu University School of Medicine, Hamamatsu, Japan
}

\section{Keywords}

Autoimmune thyroid disease $\cdot$ Lymphoproliferative disorder $\cdot$ Methotrexate $\cdot$ Thyroid lymphoma $\cdot$ Thyroid nodule

Patients with rheumatoid arthritis (RA) who are treated with methotrexate (MTX) occasionally develop lymphoproliferative disorders (LPDs) $[1,2]$. MTX withdrawal often induces prompt regression of LPD, rendering tissue biopsy unnecessary. Tokuhira et al. [1] have proposed the concept of clinical MTX-related LPDs (c-MTXLPDs), wherein the definition is LPD with prompt regression of clinical manifestations, including symptoms and abnormal laboratory data after MTX withdrawal. The thyroid gland has been rarely reported as one of the primary sites of MTX-associated LPDs (MTX-LPDs) [1-4]. Of note, there is little information regarding the sonographic characteristics of MTX-LPD of the thyroid gland and its natural course following MTX withdrawal.

A 65-year-old female with RA who was in remission complained of a painless nodule on the right side of her neck and consulted the Enshu Hospital. She had been treated with MTX for 25 years, and tocilizumab was initiated 4 years before the hospital visit. Palpation revealed a painless, firm, $2.5 \times 2.0-\mathrm{cm}$ nodule on the right side of her neck. Thyroid function tests revealed a euthyroid status. Anti-thyroglobulin and anti-thyroperoxidase antibodies levels were high $(1,490 \mathrm{IU} / \mathrm{mL}$ [reference range $<28$ ] and $36 \mathrm{IU} / \mathrm{mL}$ [reference range $<16$ ], respectively). Serum thyroglobulin and soluble interleukin-2 receptor levels were within their reference ranges. B-mode ultrasonography (US) revealed a hypoechoic nodule with the largest diameter of $24.5 \mathrm{~mm}$. The border was well defined, and its margin showed a broccoli-like growth pattern. Based on US findings (Fig. 1a, b), we initially suspected thyroid lymphoma [5]; however, fine-needle aspiration cytology (FNAC) findings revealed the benign cytological nature of the nodule based on morphological features of heterogeneous infiltrate of lymphoid cells (Fig. 1c, d). Subsequently, we suspected MTX-LPD and therefore discontinued MTX, but not tocilizumab. Because the nodular goiter showed a gradual decrease in size upon palpation, the patient preferred a periodical US follow-up rather than further clinical testing, such as lymphocyte marker study using flow cytometry by repeated FNAC and/or an immediate open biopsy. Six months later, follow-up US revealed marked decrease in lesion size (Fig. 1e). We eventually diagnosed the present case as c-MTX-LPD of the thyroid gland. The patient de- 


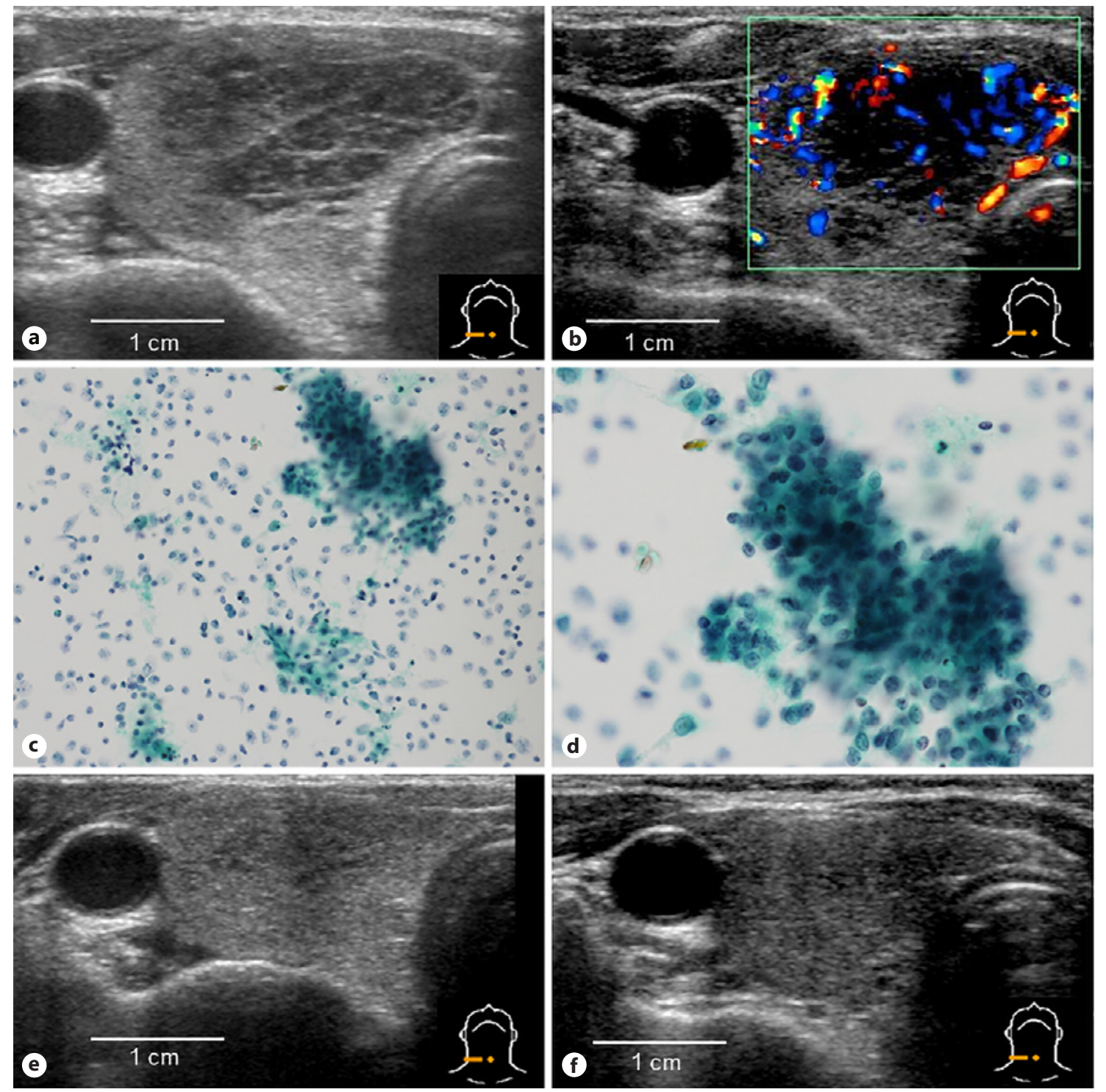

Fig. 1. Sonographic images of methotrexate-associated LPD of the thyroid gland in a 65-year-old female with an underlying autoimmune thyroid disease. a Transversal view of B-mode US showing a hypoechoic solid nodule with the enhancement of posterior echoes. Note the margin showing a broccoli-like growth pattern [5] (or a lobulated or irregular pattern according to the Thyroid Imaging, Reporting and Data System [TI-RADS] by the American College of Radiology [ACR]). The shape is wider-than-tall, and the echogenic foci are nonexistent. On the basis of these observations, the nodular lesion received a TI-RADS score of 4 (moderately suspicious). The echogenicity level of the remainder of the thyroid gland was only slightly higher than that of the adjacent muscles.

veloped no recurrence during 24 months of follow-up (Fig. 1f).

Herein we report an interesting case of a c-MTX-LPD in which sonographic findings initially suggested thyroid b Color Doppler US showing increased intranodular vascularity. c FNAC revealing small tissue fragments and numerous lymphocytes in the background (Papanicolaou stain, $\times 20$ ). d Higher magnification of a tissue fragment showing copious infiltrates of a mixed population of lymphocytes (Papanicolaou stain, $\times 40$ ) that suggests benign lymphocytic thyroiditis; however, mucosa-associated lymphoid tissue lymphoma could not be ruled out. e Followup US 6 months after MTX withdrawal showing marked decrease in lesion size. $\mathbf{f}$ US 24 months after MTX withdrawal showing that the hypoechoic lesion is almost disappeared, although the heterogeneity remains possibly due to underlying chronic thyroiditis. lymphoma and follow-up US revealed remission after discontinuation of MTX. Using follow-up computed tomography, two prior studies reported remission of thyroid lesions after MTX withdrawal in patients with RA [3, 
4]. To the best of our knowledge, this is the first reported case of an MTX-LPD of the thyroid gland in which repeated US findings are described in detail. Although further studies are required in a larger patient population, careful preoperative examination could be recommended for patients receiving MTX treatment for hypoechoic thyroid lesions that may be suspected to be thyroid lymphoma based on sonographic findings.

\section{Statement of Ethics}

All procedures followed were in accordance with the ethical standards of the responsible committees on human experimentation (institutional and national) and with the Helsinki Declaration of 1964 and later versions. Written informed consent for publication of clinical details and images was obtained from the patient.

\section{Disclosure Statement}

The authors have no conflicts of interest to declare.

\section{Funding Sources}

No specific funding was obtained for the present study.

\section{Author Contributions}

M.T. and Y.G. contributed to the acquisition of data and drafted the original manuscript. K.K., T.S., and Y.O. were involved in reviewing and editing the manuscript. K.O. is the corresponding author and organized the study.

\section{References}

1 Tokuhira M, Tamaru JI, Kizaki M. Clinical management for other iatrogenic immunodeficiency-associated lymphoproliferative disorders. J Clin Exp Hematop. 2019;59(2):7292.

2 Aoshima M, Nagayama K, Takeshita K, Ajima H, Orikasa S, Iwazaki A, et al. Methotrexate-associated lymphoproliferative disorder with hypopituitarism and central diabetes insipidus. Endocrinol Diabetes Metab Case Rep. 2019 Oct;2019:EDM190082.
3 de Mast Q, Haverman J, Netten PM, Sinnige HA. Remission of a primary thyroid lymphoma after methotrexate withdrawal. Clin Endocrinol (Oxf). 2006 Jun;64(6):716-7.

4 Watanabe K, Kajiwara K. Spontaneous remission of a large thyroid tumour. CMAJ. 2016 Apr;188(6):447.

5 Ota H, Ito Y, Matsuzuka F, Kuma S, Fukata S, Morita S, et al. Usefulness of ultrasonography for diagnosis of malignant lymphoma of the thyroid. Thyroid. 2006 Oct;16(10):983-7. 